[0212-7199 (2005) 22: 7; pp 326-328] ANALES DE MEDICINA INTERNA Copyright $@ 2005$ ARAN EDICIONES, S.L.

An. MED. INTERNA (Madrid) Vol. 22, N. ${ }^{\circ} 7$, pp. 326-328, 2005

\title{
Taponamiento cardiaco por quiste hidatídico pericárdico
}

\author{
M. SOLANO REMÍREZ, M. A. URBIETA ECHEZARRETA, M. T. ÁLVAREZ FRÍAS, \\ C. GONZÁLEZ ARENCIBIA, B. LLORENTE DÍAZ
}

Medicina Interna. Hospital de Navarra. Pamplona

CARDIAC TAMPONADE CAUSED BY HYDATID PERICARDITIS

\begin{abstract}
RESUMEN
Presentamos el caso de una mujer sin antecedentes personales de interés que debuta con insuficiencia cardiaca con clínica de taponamiento cardiaco por pericarditis hidatídica secundaria a fistulización a traves del diafragma por rotura de quista hidatídico localizado en hígado. La hidatidosis cardiaca es poco frecuente presentado una incidencia según series entre el 0,2-2\% sobre el total de infestación en humanos por Echinococcus, siendo la afectación pericárdica infrecuente. Es por ello que realizamos revisión de su etiopatogenia, presentación clínica, diagnósticos de elección y tratamientos recomendados.
\end{abstract}

PALABRAS CLAVE: Echinococcus. Pericarditis. Taponamiento pericárdico. Albendazol. Periquistectomía.

\begin{abstract}
We present the case of a woman with no previous clinical history of disease, that debuted with acute heart failure with symptoms of cardiac tamponade from hydatic pericarditis as a result of a fistula across the diaphragm secondary to a hidatidic cyst rupture in the liver. Cardiac hydatidosis is rare with an incidence in some series betweem $0.2-2 \%$ in humans infested with Echinococcus, affectation of the pericardia being rare. For this reason we present a revision of its pathogenesis, clinical presentation, diagnosis and recommended treatment.
\end{abstract}

KEY WORDS: Echinococcus. Pericarditis. Pericardial tamponade. Albendazol. Periquistectomy.

Solano Remírez M, Urbieta Echezarreta MA, Álvarez Frías MT, González Arencibia C, Llorente Díaz B. Taponamiento cardiaco por quiste hidatídico pericárdico. An Med Interna (Madrid) 2005; 22: 326-328.

\section{INTRODUCCIÓN}

La parasitación por echinococcus tiene una distribución mundial presentando zonas endémicas. El reservorio principal lo constituyen los perros y otros animales que se alimentan de vísceras infestadas de animales muertos. La afectación humana se realiza por ingesta de huevos que posteriormente invaden la circulación sanguínea infestando diferentes órganos siendo los más frecuentemente afectados el hígado y el pulmón. Los quistes hidatídicos cardiacos constituyen el 0,5-2\% de todos los quistes hidatídicos en humanos. La afectación pericárdica es infrecuente, siendo la causa más frecuente de su afectación la complicación por ruptura de quistes hepáticos. La clínica por afectación pericárdica más frecuente es la derivada por compresión. El diagnóstico se realiza por imagen (TAC y/o RMN) y ecocardiograma debiéndose realizar diag- nóstico diferencial con tumores cardiacos. El diagnóstico precoz es importante por el alto riesgo que conlleva dicha patología. El tratamiento de elección es quirúrgico.

\section{CASO APORTADO}

Se trata de una mujer de 81 años sin antecedentes de interés que refiere disnea progresiva hasta hacerse de reposo sin fiebre termometrada, acompañada de taquipnea importante con uso de musculatura accesoria, ortopnea 3 almohadas, edemas en EEII y dolor torácico tipo opresivo. En la analítica destaca la presencia de leucocitosis $28.800 / \mathrm{mm}^{3}$ con $3 \%$ cayados, pO2 56\%, SatO2 84\%. En la exploración clínica ingurgitación yugular marcada $(5 \mathrm{~cm})$, edemas en EEEII hasta rodillas, taquipnea a $28 \mathrm{rpm}$, crepitantes hasta campos medios, hepatomegalia de $6 \mathrm{~cm}$ y tonos rítmicos con tonos apagados. En el ECG destaca un pobre voltaje en derivaciones precordiales y S-T

Trabajo aceptado: 11 de enero de 2005 


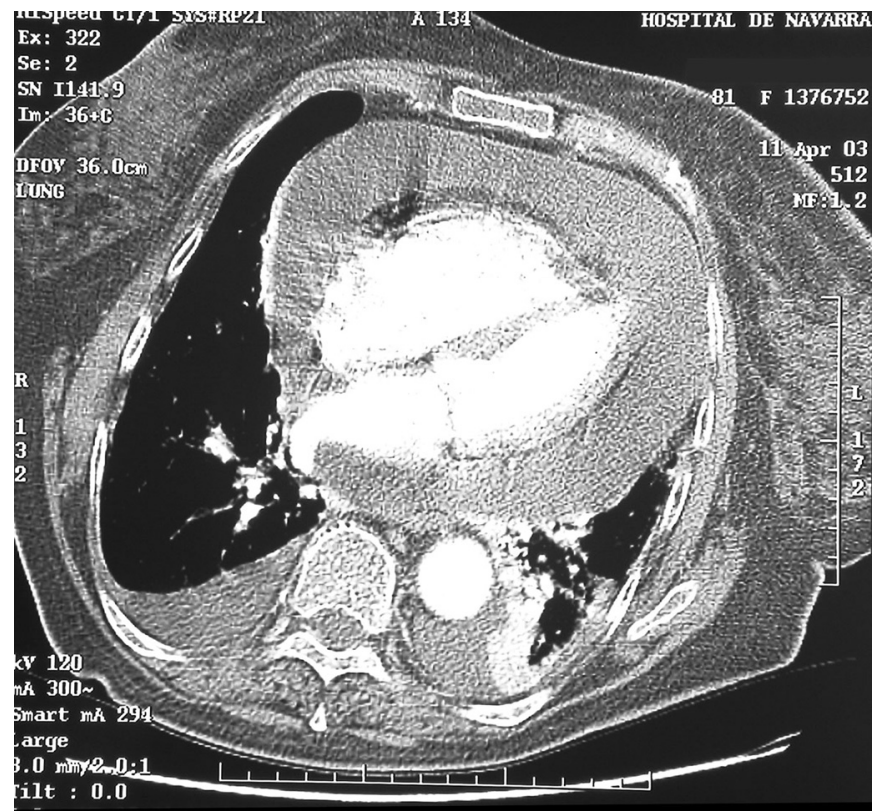

Fig. 1.

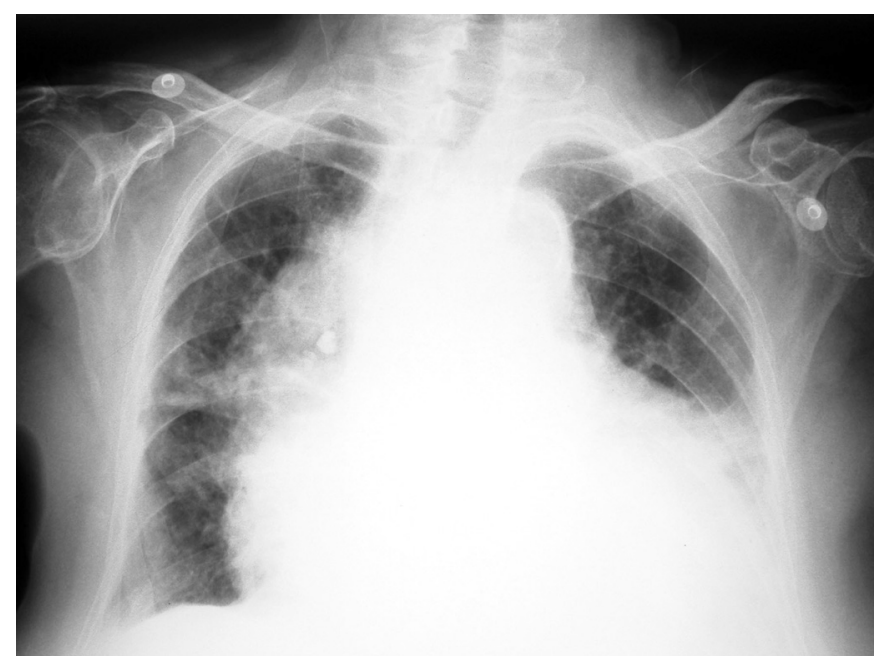

Fig. 2.

elevado de V4-V6 y cara inferior. La imagen de la radiografía de tórax refleja cardiomegalia con imagen en bota y pinzamiento costofrénico bilateral (Fig. 2). Se realiza TAC torácico que demuestra lesión quística hepática grande localizada en lóbulo izquierdo con extensión a pericardio (Fig. 1). Dada la situación clínica se ingresa en UCI requiriendo intubación orotraqueal (IOT) y soporte hemodinámico, donde, bajo anestesia local, se realiza incisión en hipocondrio izquierdo drenándose $1.200 \mathrm{cc}$ de material purulento conteniendo membranas hidatídicas; así mismo se coloca drenaje pericárdico obteniéndose material de características hemático-purulentas y vesículas. Tras presentar mejoría hemodinámica y respiratoria posterior se decide intervención bajo anestesia general realizándose laparotomía, objetivándose quiste hidatídico hepático que destruye diafragma y comunica con pericardio; se realiza ventana subxifoidea y periquistectomía subtotal con cierre de bocas vías biliares, dejándose drenaje pericárdico y $2 \mathrm{JP}$ (subfrénico y en lecho hepático). La evolución posterior fue favorable.

\section{DISCUSIÓN}

La Echinococcosis es una parasitación de distribución mundial $(1,2)$; es endémida en América del Sur, África del Norte, Europa meridional, norte y oeste del Canadá, Australia, Nueva Zelanda y zona del Valle del Misissippi y estado de Alaska en EE.UU. El agente causante es un cestodo de la familia del Echinococcus que parasita sobre todo a perros y otros cánidos (lobos, zorros) que constituyen el huesped definitivo y como huesped intermediario algunos herbívoros (ovejas, vacas, cabras y camellos). Se trasmite al hombre por contacto directo con heces infectadas y por la ingestión de huevos con los alimentos infectados. En el intestino humano los huevos se transforman en embriones u oncospheras, penetrando en la mucosa e incorporándose a la circulación sanguínea, alcanzando las vísceras que sufrirán la infestación, en forma de quistes. El periodo de incubación es largo. Se observa a cualquier edad con una cierta preferencia para el sexo femenino. Los órganos más frecuentemente afectados son el hígado (50-79\%) y el pulmón (20\%); otros (10\%) como los músculos, huesos, riñones, cerebro son más infrecuentemente infestados (3); el corazón constituye una infestación primaria rara, aproximadamente $0.2-2 \%$ de todos los casos de hidatidosis (8); siendo el septum intraventricular y el VI las localizaciones primarias cardiacas más frecuentes (4); aún es más raro la afectación del pericardio, constituyendo la equinococcosis una causa rara de afectación pericárdica produciendo taponamiento cardiaco (5). Suelen asociarse a la presencia de quistes extracardiacos $(9,11)$. La mayoría de las infecciones por echinococcus son asintomáticas, siendo la clínica más frecuente la derivada de la obstrucción de vías o la disfunción del órgano afectado; una complicación grave la constituye la ruptura del quiste y la salida del contenido a la circulación sanguínea pudiendo producir un cuadro grave de anafilaxia.

La causa más frecuente de pericarditis es la rotura a pleura y pericardio de quistes abdominales, sobre todo hepáticos, por perforación diafragmática (9-11). Los síntomas predominantes son los secundarios al taponamiento: disnea, dolor torácico $(15,17)$ El diagnostico se realiza por ecocardiograma $(6,7,16)$ y por técnicas de imagen como la TAC y/o RMN $(8,12)$; analíticamente con Ac específicos frente a equinococccus mediante ELISA e inmunoblot (sensibilidad del 80-100\%), siendo característico la presencia de eosinofilia (59\% de casos) en sangre periférica. El diagnóstico diferencial debe realizarse con tumores de origen cardiaco, formaciones quísticas tipo teratomas, quistes congénitos del pericardio, quistes secundarios a hematomas intrapericárdicos o degeneraciones quísticas de tumores (16). Es importante conocer si la zona es endémica para equinococcosis. Ocasionalmente, como complicación, puede producirse sobreinfectación con pericarditis purulenta secundaria.

El tratamiento de elección es quirúrgico $(13,14,18)$. Con objeto de disminuir el riesgo de siembra peritoneal en caso de producirse rotura accidental del quiste durante la intervención quirúrgica se aconseja administrar antes y después de la cirugía albendazol (10-15 mg/kg) y esterilizando el quiste con solución de $\mathrm{CINa}$ al 20\%; posteriormente se debe continuar tratamiento con albendazol a las mismas dosis (10-15 $\mathrm{mg} / \mathrm{kg} /$ día) durante 3 meses (según unos autores descansando una semana de cada cuatro, y según otros de forma continua) coadministrando, opcionalmente, praziquantel. Se debe reali- 
zar profilaxis en áreas endémicas mediante tratamiento a los perros con praziquantel $5 \mathrm{mg} / \mathrm{kg}$ mensual.

Podemos concluir que, siendo la parasitación por Echinococcus de distribución mundial, la afectación cardiaca es rara y más infrecuente la pericárdica. El diagnóstico diferencial, en zonas endémicas, debe realizarse con los tumores cardiacos.
El diagnóstico mediante pruebas de imagen resulta sencillo y rápido. El tratamiento de elección es quirúrgico mediante periquistectomía realizando previamente esterilización del quiste y con albendazol antes y después de la intervención, continuando durante al menos 3 meses asociando, de forma opcional, praziquantel.

\section{Bibliografía}

1. Tiseo D, Borrelli F, Gentile I, Benassai G, Cuarto G, Borgia G. Cystic echinococcosis in humans: our clinic experience. Parassitologia 2004; 46: 45-51.

2. Bhatia G. Echinococcus. Semen Respir Infect 1997; 12: 171-86.

3. Vasen W, Florentino RM. Clinical manifestations and late complications of the hepatic hydatid cyst: report of 4 cases. Acta Gastroenterol Latinoam 2004; 34: 83-7.

4. DiBello R, Abo JC, Borges UL. Hydatid constrictive pericarditis. A new case and review of the literature. J Thorac Cardiovasc Surg 1970; 59: $530-2$

5. Shojae S, Hutchins GM. Echinococcosis complicated by purulent pericarditis. Chest 1978; 73: 512-4.

6. Dighiero J, Canabal EJ. Echinococcus disease of the heart. Circulation 1958; 17: 127-132.

7. Rey M, Alfonso F, Torrecilla EG, McKenna WJ, Balaguer J, Alvarez L, Rabago P, Rabago G, Nihoyannopoulos P. Diagnostic value of twodimensional echocardiography in cardiac hydatid disease. Eur Heart J 1991; 12: 1300-7.

8. Noah MS, el Dinn Hawas N, Joharjy I, Abdel-Hafez M. Primary cardiac echinococcosis: report of two cases with review of the literature. Ann Trop Med Parasitol 1988; 82: 67-73.

9. Iglesias LF, Zabala y Morales M, Marcos G, González Eguaras M, Vega J, Vaccari O, et al. Pericarditis secondary to the rupture of a hydatid cyst. Rev Esp Cardiol 1999; 52: 135-8.

10. Karadede A, Ulgen MS, Temamogullari AV, Toprak N. A complicated

case of pericardial hydatid cyst manifesting as constrictive pericard. Can J Cardiol 2000; 16: 673-6

11. Ahuja SR, Karande S, Koteyar SR, Kulkarni MV. Hepatic hydatid cyst rupturing into sub-diaphragmatic space and pericardial cavi. J Postgrad Med 2001; 47: 37-9.

12. Saidi F. Images in clinical medicine: echinococcal cyst of the pericardium. N Engl J Med 1995; 333: 1572.

13. Djoshibaev S, Kudaiberdiev T, Maralov A, Shabraliev S, Djooshev K, Halikov UM, Yankovskaya L. Surgical treatment of isolated cardiac echinococcosis: report of five cases. Anadolu Kardiol Derg 2003; 3: 137-43.

14. Buttenschoen K, Schort P, Reuter S, Kern P, Carli Buttenschoen D, Henne-Bruns D. Surgical treatment of hepatic infection of Echinococcus granulosus. Z. Gestroenterol 2004; 42: 1101-8.

15. Drissa H, Zayani Z, Boussaada R, Zaouali RM. Cardiac hydatid cyst (report of 8 cases). Tunis Med 2001; 79: 633-7

16. Antonini-Canterin F, Piazza R, Ascione L, Pavan D, Nicolosi GL. Value of transesophageal echocardiography in the diagnosis of compressive, atypically located pericardial cysts. J Am Soc Echocardiograf 2002; 15: 192-4.

17. Birincioglu CL, Bardakci H, Kucuker SA, Ulus AT, Arda K, Yamak B, Tasdemir O. A clinical dilemma: cardiac and pericardiac echinococcosis. Ann Thorac Surg 1999; 68: 1290-4.

18. Mónaco M, Mondillo B, Galletti G, Capuccio S, Monici D, Nunnari F, et al. Surgical treatment of hydatic cysts. G Chir 2004; 25: 224-6. 